\title{
INTEGRATION OF UPC-UAB FAST SOLVERS INTO THE ANTENNA SIMULATION SOFTWARE OF UNEX-UPM, KUL, UPV AND EPFL ACE PARTNERS
}

\author{
A. Heldring ${ }^{(1)}$, J.M. Rius ${ }^{(1)}$, J.M. Tamayo ${ }^{(1)}$, J.Rubio ${ }^{(2)}$, J.Zapata ${ }^{(3)}$, V.Volski ${ }^{(4)}$, G.Vandenbosh ${ }^{(4)}$, \\ M.Mattes $^{(5)}$, A. Valero $^{(6)}$, J.Parrón ${ }^{(7)}$ \\ (1) AntennaLab, Dept. of Signal Theory and Communications, \\ Universitat Politècnica de Catalunya (UPC), Spain, rius@tsc.upc.edu \\ (2)Dept. de Tecnología de Computadores y de las Comunicaciones, Universidad de Extremadura (UNEX), Spain. \\ (3) Dept. de Electromagnetismo y Teoría de Circuitos, Universidad Politécnica de Madrid (UPM), Spain. \\ (4) Katholieke Universiteit Leuven (KUL), Belgium \\ (5)Laboratory of Electromagnetics and Acoustics, Ecole Polytechnique Fédérale de Lausanne (LEMA-EPFL), Switzerland. \\ (6) Dpt. of Communications, Universitat Politècnica de Valencia (UPV), Spain \\ (7) Antenna and Microwave Systems Group, Dpt. of Telecommunication and System Engineering, Universitat Autònoma de \\ Barcelona (UAB), Spain.
}

Keywords: antenna analysis, MoM, direct solution.

\begin{abstract}
Software integration between Antennas Centre of Excellence (ACE) partners is one of the main activities of the Antenna Software Initiative (ASI) work package. This paper will present the results of integrating the $\mathrm{CBD}$ fast direct solver for large systems of equations, developed at UPC AntennaLab, into the antenna simulation codes of UPMUNEX, KUL, UPV and EPFL ACE partners. A non-ACE group (UAB) is closely cooperating with UPC in the development of fast solvers.
\end{abstract}

\section{Introduction}

In recent years, a wide range of methods has been developed for accelerating the iterative solution of the discretized electromagnetic integral equations [2]. However, in some cases direct instead of iterative methods are necessary, either because the linear system is poorly conditioned or because there are many different excitation vectors. UPC-UAB group has developed both iterative and direct solvers for very large systems of equations. They are applicable to Method of Moments (MoM) discretizations with any sub domain basis function set and with most commonly used Green's functions in the Integral Equation. This paper presents the first results obtained by the partner-groups within ACE using the fast direct solver called Compressed Block Decomposition (CBD) developed by UPC-UAB [4].

The CBD is presently being integrated into the codes of KUL and LEMA-EPFL and has already been successfully integrated with the MoM code of UPV and with a full-wave software, the Finite Array GSM Code, which has been developed by the ECAM group from UPM and UNEX for the analysis of finite arrays of elements which can be described by means of spherical waves such as apertures, horns, monopoles, cavity-backed patch antennas or DRAs. The results of this integration are presented in detail in another Eucap paper [8]. The integration of CBD into existing codes within ACE is addressed in sections. 3 and 4.

\section{CBD}

The CBD direct solver is a powerful method that can, on the one hand, accelerate significantly the solution of small problems (a few thousands of unknowns) with respect to traditional direct LU decomposition, and on the other hand, it brings moderately large problems (up to several tens of thousands of unknowns) within reach of a typical modern desk-top personal computer. Another noteworthy advantage of the method is the very low marginal cost per excitation vector, as illustrated by the results presented in this paper. Being an entirely algebraic algorithm, $\mathrm{CBD}$ can be used to solve any linear system, not only those resulting from MoM or even electromagnetics simulation problems. Furthermore, it is very easy to integrate with most existing MoM (or other) codes. The present UPC-UAB tool only needs as input the user-generated coefficient matrix, in a block-by-block format, and the independent vectors. The matrix blocks can be chosen absolutely arbitrarily by the user, but the method works best if

- The blocks are approximately equal in size, and not too many or too few. The exact number of blocks is not critical but an experimental rule of thumb for the number of groups is given by $N^{1 / 2} / 3$, where $N$ is the total number of unknowns.

- The blocks represent interactions between geometrically grouped basis-functions, such that, when two groups are far apart with respect to their size, the group-to-group interaction is low-rank (see [4]). 
It is up to the user to implement the above rules to the extent that their code allows, but the geometrical sub-division of the problem into groups is a procedure that is well known from the various fast iterative methods [2] and should not be a barrier to the implementation. The output of the tool is the surface current vectors, from which the user can compute the desired results (far field, RCS, etc.). The procedure is shown schematically in Fig. 1.

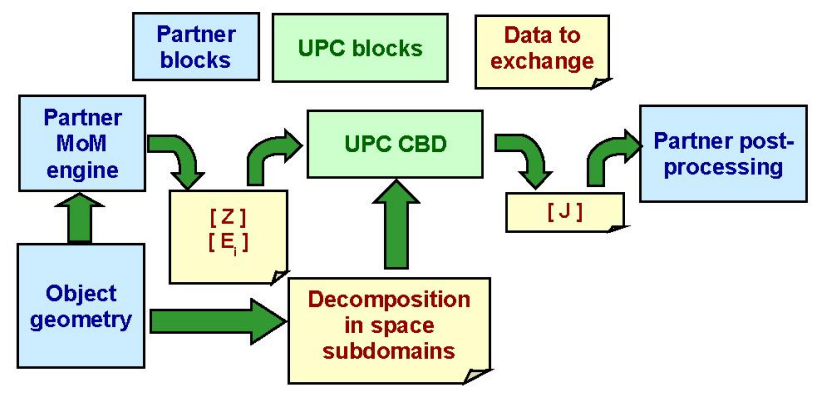

Figure 1: Integration of CBD solver.

The CBD provides an efficient way to solve the linear system resulting from MoM discretization of electromagnetic problems. However, the entire impedance matrix must be generated, and after the blocks are passed to the CBD tool, they are compressed by way of thresholded singular value decomposition (SVD). This means that the original block is decomposed into the usual SVD factors $U, S$ and V, and all lines and columns corresponding to singular values below the threshold with respect to the largest singular value are eliminated. While providing optimum compression, the SVD is a notoriously expensive algorithm. Altogether, the generation and compression of the impedance matrix is presently typically the bottleneck of the tool in terms of computational time.

At UPC-UAB, an efficient implementation of the Matrix Decomposition Algorithm (MDA) [5,7] has been developed that highly accelerates the compression procedure, avoiding entirely the need to construct the full original matrix blocks. The next integration step within the ACE framework will be to release a version of MDA to the partners that can be integrated with their MoM code. Another method to achieve this aim presently under consideration is the Adaptive Cross Approximation (ACA) [1].

The ACE partners of KUL and LEMA-EPFL are presently adapting their codes to work with CBD. The partners of the ECAM group from UPM and UNEX and the partners from UPV have successfully integrated the CBD with their code. Some results of this integration are presented in the next sections.

\section{Conformal spherical patch array}

At ACE partner UPV the CBD tool has been successfully integrated with a MoM code for scattering and radiation from metallic surfaces. The integration was particularly simple in this case since the partner's code and the CBD tool are implemented in the same environment (Matlab). The code was tested on an ACE benchmark problem (provided by Chalmers University of Technology in the framework of ACE work package 1.1-2), named Conformal Spherical Patch Array [6]. The structure is shown in Fig. 2 and the dimensions are given in Table 1.

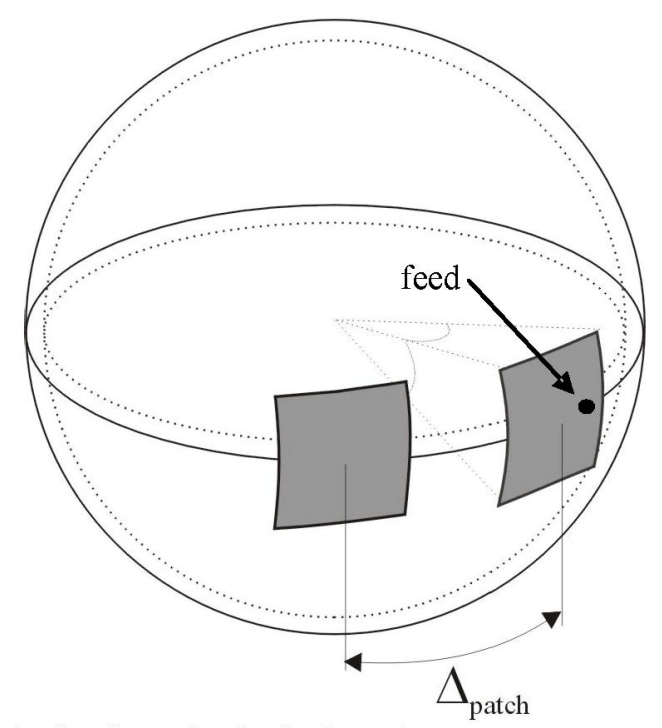

Figure 2: Conformal spherical patch array

\begin{tabular}{|l|c|}
\hline \multicolumn{1}{|c|}{ Parameter } & Size \\
\hline Sphere radius & $18.7 \mathrm{~cm}$ \\
Distance patches - sphere & $0.52 \mathrm{~cm}$ \\
Patches side length & $5.1 \mathrm{~cm}$ \\
$\Delta_{\text {patch }}$ & $10.25 \mathrm{~cm}$ \\
\hline
\end{tabular}

Table 1: Dimensions of structure from Fig. 2.

Both the sphere and the square patches are considered to be PEC surfaces. The patches are square and conformal to the sphere surface. The working frequency is $2.5 \mathrm{GHz}$. One patch is fed by a $50 \Omega$ coaxial at the spot indicated in Fig. 2. The entire structure was discretized using 11542 RWG basis functions. Fig. 3 shows the discretized model of the feed. 
The results of the CBD algorithm were compared with those of the direct, unapproximated blocked LU decomposition which was executed out-of-core. The experiment was carried out on a $\mathrm{PC}$ with an Intel Pentium 4-processor at $3.2 \mathrm{GHz}$ with $2.0 \mathrm{~GB}$ of RAM under Ms Windows XP SP2. Both algorithms used a subdivision of the structure into 36 blocks; all of approximately the same size and subdivided according to the above-mentioned geometrical criterion (which obviously only affects the CBD performance). Table 2 summarizes the respective computational costs.

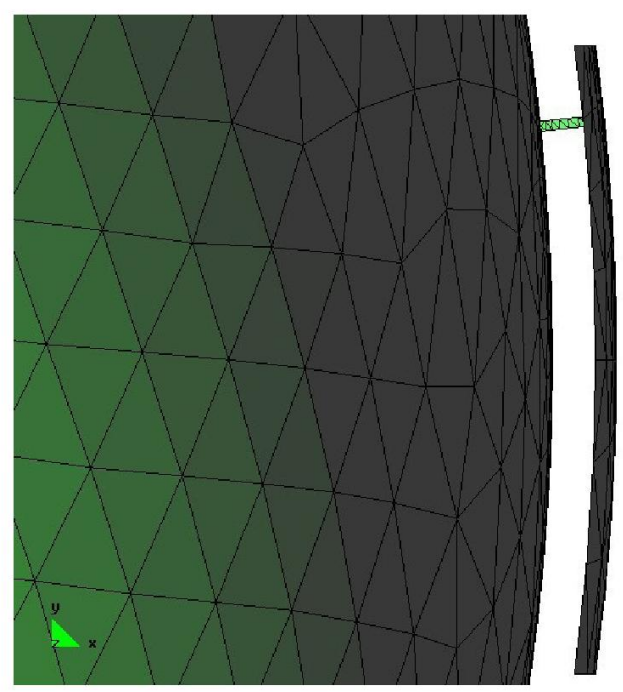

Fig. 3: Detail of the discretized spherical patch array, showing one patch and the feeding probe.

\begin{tabular}{|c|c|c|}
\hline & Block-LU & CBD \\
\hline Constructing the matrix & $3660 \mathrm{~s}$ & $4890 \mathrm{~s}$ \\
\hline Decomposition & $4974 \mathrm{~s}$ & $427 \mathrm{~s}$ \\
\hline $\begin{array}{c}\text { Solving for one } \\
\text { excitation vector }\end{array}$ & $73 \mathrm{~s}$ & $0.3 \mathrm{~s}$ \\
\hline $\begin{array}{c}\text { Total size of } \\
\text { decomposition }\end{array}$ & $2 \mathrm{~GB}$ & $320 \mathrm{MB}$ \\
\hline
\end{tabular}

Table 2: Timing and storage for CBD compared with straightforward block LU decomposition for an $N=11542$ problem.

First of all, one notices that the matrix construction time is longer for CBD than for blocked LU. This is because for $\mathrm{CBD}$, the blocks need to be SVD compressed as explained in the previous section. The difference in time corresponds exactly to the SVD compression. The threshold for the SVD compression, as well as for the re-compression steps inside the $\mathrm{CBD}$ algorithm was set to $0.01 \%$ for this experiment. With the MDA package developed at UPC-UAB, to be made available to the $A C E$ partners, the total matrix construction time is typically smaller than the decomposition time. First test results at UPC show that the same is true for $\mathrm{ACA}$.
The decomposition time is more than 10 times less for CBD than for direct $\mathrm{LU}$ and the reduction in storage requirement is considerable as well. The most striking difference is in the solution by back-substitution, which is more than 200 times faster with CBD. For this particular experiment there was only one independent vector to be solved for, but it is evident that with many excitation vectors (computing monostatic RCS for example), the use of CBD pays off seriously.

Fig. 4 shows the surface current density as computed by CBD (the image is indistinguishable from that of the direct solution).

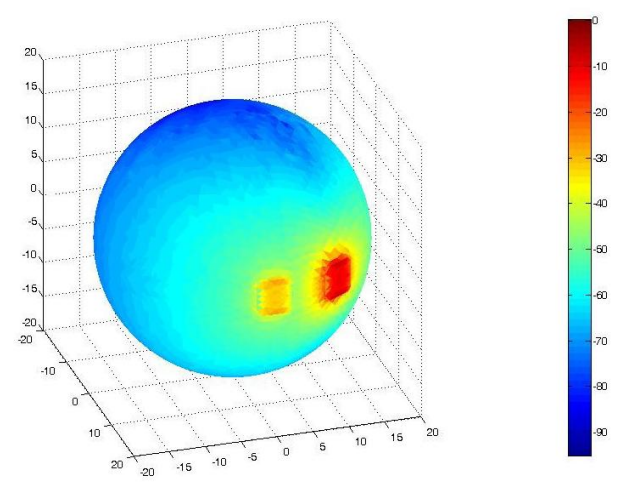

Fig. 4: Surface current density distribution on spherical patch array, computed with CBD.

The CBD accuracy was validated by comparing the radiation patterns as computed by CBD with those from the direct solution. As Fig. 5 shows, the patterns correspond very well.

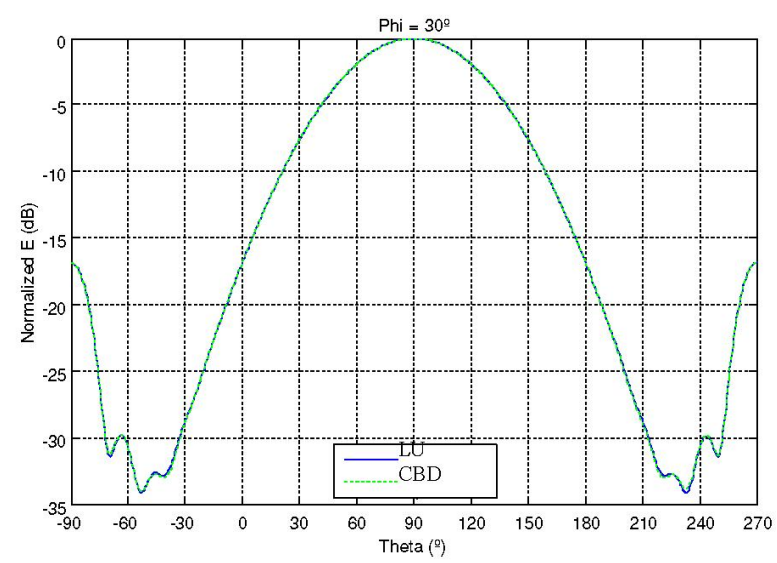




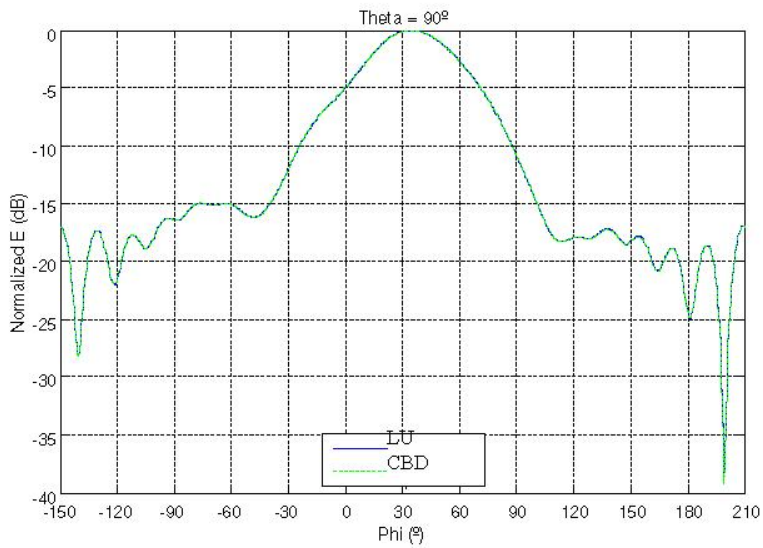

Figure 5: Radiation patterns of spherical patch array.

\section{Large array of square patches}

The ECAM group from UPM and UNEX has successfully integrated the $\mathrm{CBD}$ with their powerful full-wave software, the Finite Array GSM Code for the analysis of finite arrays of elements which can be described by means of spherical waves [3]. The code has been applied to an array of $71 \times 71$ elements, each of which is characterised by 15 modes. This results in a linear system of 75615 unknowns, much too large for direct solution. In order to prepare the problem for $\mathrm{CBD}$, the elements were grouped into 80 groups, most of them consisting of $8 \times 8$ elements (with a few smaller residual groups) and the matrix blocks representing interactions between distant groups were compressed using SVD with a threshold of $0.1 \%$. The resulting representation of the linear system is presented to the CBD algorithm, which performs the decomposition and the solution for any number of excitations. Table 3 shows the computational resources needed for this procedure (This experiment was carried out on a PC with a $2.2 \mathrm{GHz}$ processor and $8 \mathrm{~GB}$ of RAM). The details of this analysis are presented in [8].

\begin{tabular}{|c|c|c|}
\hline Build time & CBD time & Compressed matrix size \\
\hline 3h. $48 \mathrm{~min}$. & $1 \mathrm{~h} .42 \mathrm{~min}$ & $5.5 \mathrm{~GB}$ \\
\hline
\end{tabular}

Table 3: Compressed matrix build time, CBD time and matrix size for a $71 \times 71$ patch array with 75615 unknowns.

The solution by back-substitution for one excitation vector for this problem took only 2.7 seconds. This again illustrates the fact that the CBD method is particularly useful when solving for multiple excitations: once the decomposition is done, the cost per excitation vector is minimal.

This example also illustrates the observation made in the introduction that the initial matrix compression, when done by straightforwardly creating and SVD-compressing the matrix blocks, may turn out to be the most expensive step in the algorithm, underscoring the need for a fast compression method like MDA or ACA.

This problem was too large to validate the $\mathrm{CBD}$ result through a comparison with a direct solution without approximations. In [8] the validation is obtained by a comparison with the result for an infinite array, showing good correspondence. Here we validate the $\mathrm{CBD}$ by a comparison of the results for a problem that ECAM was able to solve directly, without the aid of CBD. This was a $25 \times 25$ array of the same elements, a problem with 9375 unknowns. Fig. 6 shows the comparison of the computed radiation patterns when the central element is excited. The results are virtually indistinguishable.

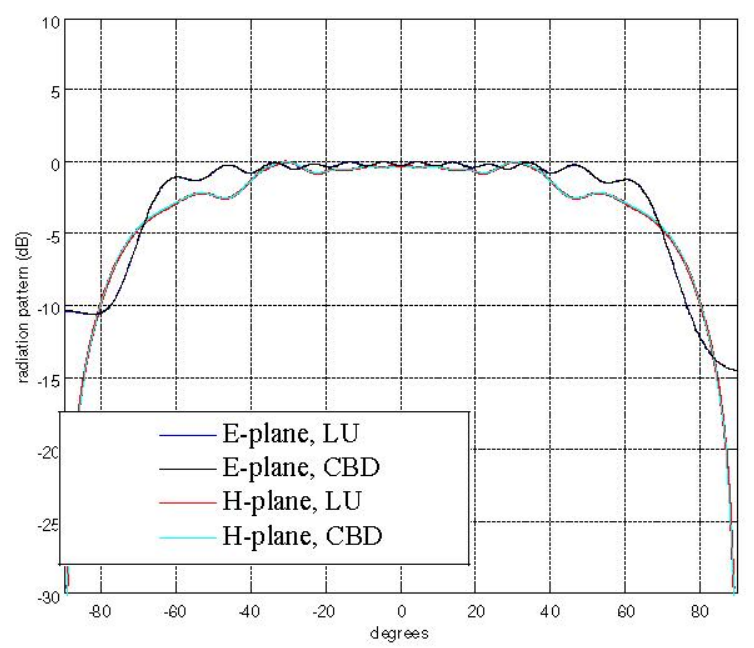

Figure 6: Central active element field pattern of $25 \times 25$ element array computed with CBD and with LU.

\section{Conclusions}

A new fast direct solution code, the Compressed Block Decomposition algorithm (CBD), has been released to the ACE partners by UPC. It is presently being integrated with the electromagnetic engines of two ACE partners, KUL and EPFL. Two other partners, UPV and UPM (in collaboration with UNEX), have already successfully implemented the CBD in their electromagnetics code. Some results from the latter two groups have been presented in this paper

\section{Acknowledgements}

This work was supported by the European Commission under contract FP6-IST-2006-026957, ACE-2, Antenna Centre of Excellence, and by the Spanish "Comisión Interministerial de 
Ciencia y Tecnología" (CICYT) through the "Ramón y Cajal" Programme and projects TEC2006-13248-C04-02/TCM and TEC2004-00950/TCM.

\section{References}

[1] M. Bebendorf, "Approximation of boundary element matrices," Numer. Math. 86: 565:589 (2000)

[2] W. C. Chew, J-M. Jin, C.-C. Lu, E. Michielssen, and J. M. Song, "Fast Solution Methods in Electromagnetics," IEEE Transactions on Antennas and Propagation, Vol. 45, No. 3, pp. 533-543, March 1997.

[3] A. González, J.A. Encinar and J. Zapata, "Radiation Pattern Computation of Cavity-Backed and Probe-Fed Stacked Microstrip Patch Arrays," IEEE Trans. on Antennas Propagation, volume 48 no. 4, pp. 502-509, April 2000.

[4] A. Heldring, J.M. Rius, J.M. Tamayo, E. Ubeda and J. Parrón, "Fast Direct Solution of MoM linear system," accepted for publication in IEEE Trans. on Antennas and Propagation.

[5] E. Michielsen, and A. Boag, 'A Multilevel Matrix Decomposition Algorithm for Analyzing Scattering from Large Structures,' IEEE Trans. on Antennas and Propagation., Vol. 44, No. 8, 1086-1093, Aug. 1996.

[6] Z. Sipus, N. Burum, and J. Bartolic, "Analysis of rectangular microstrip patch antennas on spherical structures," Microwave and Optical Technology Letters, Vol. 36, pp. 276-280, Feb. 2003.

[7] J.M. Rius, J. Parrón, E. Ubeda and J.R. Mosig, 'Multilevel Matrix Decomposition Algorithm for Analysis of Electrically Large Electromagnetic Problems in 3-D,' Microwave and Optical Technology Letters, Vol. 22, No. 3, 177-182, August 199

[8] J. Rubio, A. Heldring, J. M. Rius, M.A. González, J. Zapata, "Integration of CBD Solver in the Finite Array GSM Code," European Conference on Antennas and Propagation (EuCAP 2007), Edinburgh, 11-16 November 2007 